\title{
Quantum manifestation of elastic constants in nanofilms
}

\author{
Miao Liu and Feng Liu \\ Department of Materials Science and Engineering, University of Utah, Salt Lake City, UT 84112, USA \\ E-mail: fliu@eng.utah.edu
}

Received 21 October 2013, revised 23 December 2013

Accepted for publication 23 January 2014

Published 4 March 2014

\begin{abstract}
Generally, there are two distinct effects in modifying the properties of low-dimensional nanostructures: surface effect (SE) due to increased surface-volume ratio and quantum size effect (QSE) due to quantum confinement in reduced dimension. The SE has been widely shown to affect the elastic constants and mechanical properties of nanostructures. Here, we demonstrate that QSE can also have a strong effect on the elastic constants of nanofilms from first-principles calculations. We conclude that generally QSE is dominant in affecting the elastic constants of metallic nanofilms while SE is more pronounced in semiconductor or insulator nanofilms. We also demonstrate that QSE affects the elastic constants of armchair graphene nanoribbons. Our findings have broad implications in quantum aspects of nanomechanics.
\end{abstract}

Keywords: nanofilm, nanomechanics, quantum confinement, elasticity

S Online supplementary data available from stacks.iop.org/Nano/25/135706/mmedia

(Some figures may appear in colour only in the online journal)

\section{Introduction}

Mechanical properties of nanoscale solid structures are different from their bulk counterparts. It has been demonstrated experimentally that elastic moduli change their values as a function of the size of nanostructures, such as the diameter of nanorods or thickness of nanoplates [1,2]. The general understanding is that such size dependence of elasticity has its physical origin in the elasticity variation at material surface. It is well-known that surface has a different structure from underlying bulk due to bond breaking, surface relaxation and reconstruction, which gives rise to excess surface energy and non-zero intrinsic surface stress [3-5]. Consequently, the elastic constants of surface (which may include several atomic layers $[4,5])$ are distinctively different from those of bulk. In a nanostructure, the surface-to-volume ratio continues to increase when its size decreases, so that the overall elastic constants of the nanostructure will exhibit a strong size dependence.

There have been many studies about the elastic constants of nanofilms focusing on surface effect (SE). For example, experiments showed that Young's modulus of a thin film can either increase or decrease relative to bulk when the film thickness approaches nanoscale [6, 7]. Theoretically, it is found that the surface could decrease Young's modulus down to $2 / 3$ of its bulk value from calculations using harmonic or Lennard-Jones potential approximation [8, 9]. Another calculation found that Young's modulus of thin film varies as inverse of its thickness, which could go either larger or smaller than the bulk value, based on embedded atom method (EAM) and Stillinger-Weber potential simulation [10]. EAM simulations of $\mathrm{Cu}$ film showed that $\mathrm{Cu}$ surface could become either stiffer or softer relative to bulk depending on surface and uniaxial strain orientation [11]. In general, elastic constants of nanostructures have been modeled by partitioning the structure into two parts of inner bulk and outer surface with modified surface elastic constants [11-13]. This makes the overall mechanical properties of nanostructures different from those of their bulk form. For example, the mechanical bending of nanofilms follows the modified Stoney [12] and Timoshenko [13] formula rather than the classical formula for macroscopic thick films. 
Besides the SE, there is another effect becomes increasingly prominent at the nanoscale to affect the properties of low-dimensional nanostructures, i.e., quantum size effect (QSE). When the dimension of a nanostructure is reduced to be comparable to the electron Fermi wavelength, electrons become geometrically confined giving rise to quantized electronic states that change electronic energy, which in turn modify various properties of nanostructures, such as surface energy [14], stability [15] and magnetism [16]. Therefore, it is reasonable to expect the QSE to affect the mechanical properties of nanostructures. Recent theoretical [17-19] and experimental [20] studies have indeed shown the QSE causing quantum oscillations of surface (edge) stress in nanostructures. In general, however, despite the extensive study of the SE on the elastic constants of nanostructures [1, 2, 6-13], little attention has been paid to the QSE.

Here, we demonstrate quantum manifestations of elastic constants in nanofilms induced by QSE using first-principles calculations. Using $\mathrm{Pb}, \mathrm{Al}, \mathrm{Si}$ nanofilms as prototypes, we show that their Young's modulus and Poisson's ratio can display an oscillatory dependence on the thickness of nanofilms. The main physical origin for such quantum oscillations of elastic constants is the QSE induced oscillation of electron density in the direction of confinement. Because electron Fermi wavelength is much shorter in a metal than in a semiconductor or insulator, generally the QSE is more prominent, even overwhelming the SE, in affecting the elastic constants of metal nanofilms, but less important in semiconductor and insulator nanofilms. As a special case, we additionally show similar QSE in armchair graphene nanoribbons (AGNR). It is also important to point out that previous theoretical studies [8-13] used empirical potentials which did not account for electronic effects. Consequently, the QSE on elastic constants will be missed in these earlier studies, which calls for the need of first-principles calculations as presented here.

\section{Methodology}

Our calculations are carried out using the density functional theory method as implemented in the VASP code [21] with the projector augmented wave method [22] and the PerdewBurke-Ernzerhof exchange-correlation functional [23]. As shown in figure $1(\mathrm{a}), \mathrm{Pb}(111), \mathrm{Al}(111)$ and $\mathrm{Si}(111)$ film are modeled by a supercell slab set at the theoretical bulk lattice constant of $5.04 \AA, 4.02 \AA$ and $5.47 \AA$, respectively, as the reference of strain-free state ${ }^{1}$. The slabs are separated by a vacuum thickness of $20 \AA$ in $z$-direction, sampled by a $21 \times 21 \times 1$ mesh for $\mathrm{Pb}(111)$ and $\mathrm{Si}(111)$ film, and $61 \times 61 \times 1$ mesh for Al(111) film in $k$-space. AGNRs are modeled by using similar super cell technique (figure 1(b)) with a vacuum thickness of $20 \AA$ in both $y$ and $z$ directions, sampled by a $10 \times 1 \times 1$ mesh in $k$-space. All calculations

${ }^{1}$ Following the original Gibbs' definition of 'excess' surface quantity of the dividing surface of a bulk [1], surface quantities, including those of nanostructures, should always be defined relative to bulk. For a nanofilm, one may image the film sitting on a substrate. Note that if one fully relaxes the film structure, surface quantities would become ill-defined. (a)

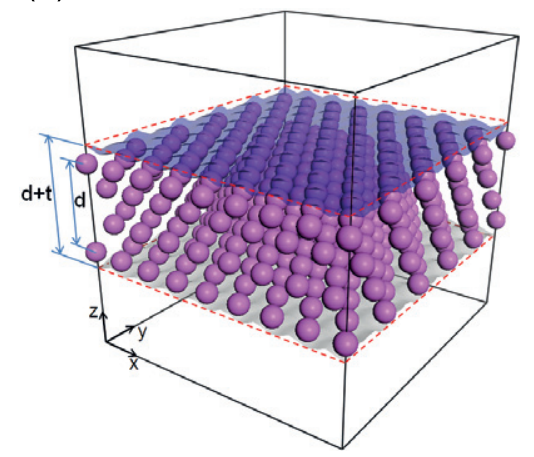

(b)

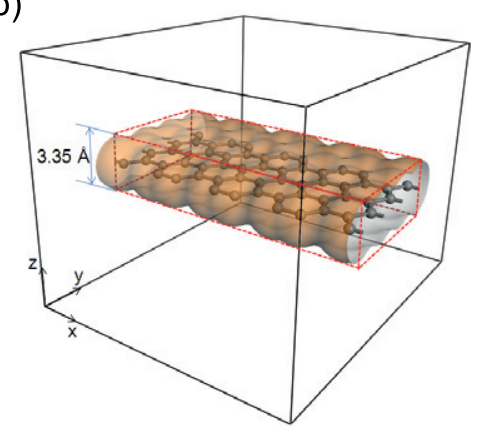

Figure 1. Schematic plot of computational supercell of (a) $\mathrm{Pb}(111)$, $\mathrm{Al}(111)$ or $\mathrm{Si}(111)$ film and (b) AGNR. Vacuum region was shown in the outer solid black box, and the thickness convention is shown in the inner dashed red box.

used a plane-wave cutoff of 1.3 times of default value and the structures are optimized until the atomic force converged to $1 \mathrm{meV} \AA^{-1}$. We extracted Young's modulus $(E)$ and Poisson's ratio $(v)$ from the calculated stress-strain relations in the linear elasticity regime as a function of film thickness or ribbon width, using an applied strain of $1 \%$ for nanofilm and $3 \%$ for $\mathrm{GNR}^{2}$. Surface and edge reconstructions are neglected to isolate the QSE. This is a reasonable approximation, because inclusion of surface reconstruction will only cause a constant shift of surface energy or surface stress, but without changing its monotonic film thickness dependence and hence without affecting our analysis of QSE that causes an oscillatory thickness dependence.

Young's modulus is conventionally defined as the ratio of uniaxial tensile stress over uniaxial tensile strain $\left(E=\frac{\sigma_{\|}}{\varepsilon_{\|}}\right)$. And Poisson ratio is defined as the ratio between biaxial transverse compressive strain over longitudinal uniaxial tensile strain $\left(v=-\frac{\varepsilon_{\perp}}{\varepsilon_{\|}}\right)$. Here, to better reveal the QSE that is induced by confinement only in the film thickness direction, we apply biaxial compressive strain $\left(\varepsilon_{\mathrm{bi}}\right)$ in the film surface $x-y$ plane (see figure 1(a) for Cartesian axes), and calculate the strain induced in-plane film stress $\left(\sigma_{\mathrm{bi}}\right)$ (note that the intrinsic surface stress in the absence of strain is subtracted) and out-of-plane responsive uniaxial tensile strain $\left(\varepsilon_{z}\right)$ in the surface normal direction. Correspondingly, we define the film Young's modulus as $E_{\mathrm{f}}=\frac{\Delta \sigma_{\mathrm{bi}}}{\Delta \varepsilon_{\mathrm{bi}}}$ and Poisson's ratio as $v_{\mathrm{f}}=-\frac{\Delta \varepsilon_{\mathrm{bi}}}{\Delta \varepsilon_{z}}$. It can be shown that our definition of biaxial

${ }^{2}$ See supplementary material (available at stacks.iop.org/Nano/25/1 35706/mmedia). 
(a)



(c)



(e)

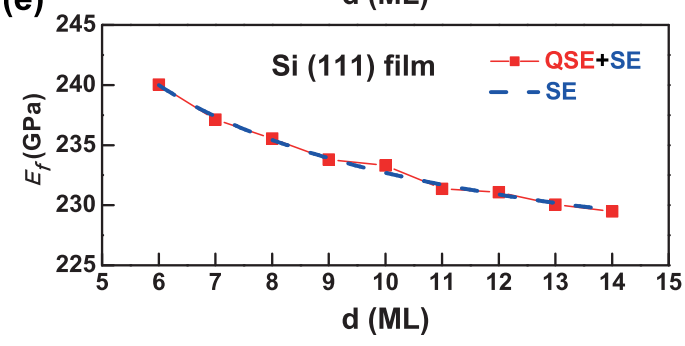

(b)

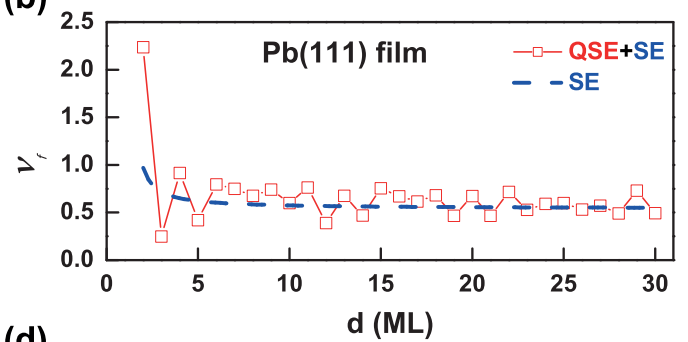

(d)

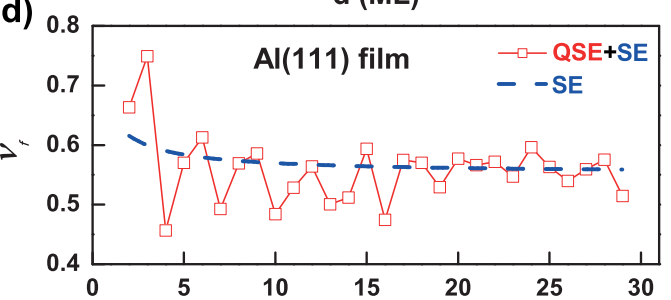

(f)

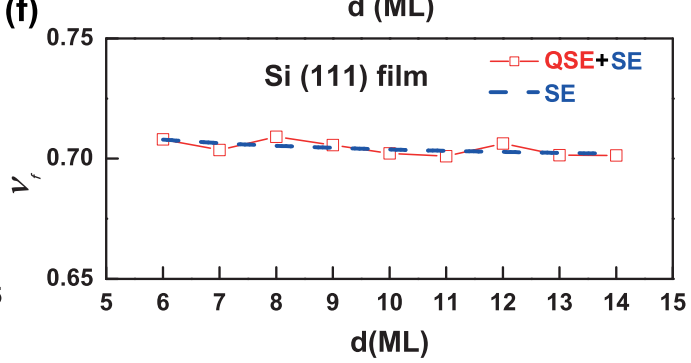

Figure 2. ((a), (c), (e)) Young's modulus and ((b), (d), (f)) Poisson's ratio of Pb(111), Al(111) and Si(111) film as a function of film thickness, respectively. The dashed blue lines show the SE fitted using equation (3).

Young's modulus and the conventional definition of uniaxial Young's modulus differ by a factor of $1 /(1-v)$. The reason for our choice is to maintain the lattice symmetry and to isolate the QSE in the calculations. For AGNRs, we can simply use the conventional definition $E_{\mathrm{r}}=\frac{\Delta \sigma_{x}}{\Delta \varepsilon_{x}}$ and $\nu_{\mathrm{r}}=-\frac{\Delta \varepsilon_{y}}{\Delta \varepsilon_{x}}$ (see figure 1(b)). Another point to be noted for systems with surface and edge is how to define their thickness and width. Here, we use the convention that the thickness (width) is set equal to the distance between the two outmost atomic planes (rows) plus one interlayer (inter-row) spacing, as show in figure 1(a) (figure 1(b)). For example, the interlayer spacing of $\mathrm{Pb}(111)$ film is $2.90 \AA$, and the interlayer spacing of AGNR is $3.35 \AA$. The same thickness values are used throughout for consistency.

\section{Results}

We first calculated the elastic constants of metallic $\mathrm{Pb}(111)$ and $\mathrm{Al}(111)$ nanofilms, which are known to exhibit strong QSE [19, 20, 24, 25]. Figures 2(a) and (b) show the calculated $E_{\mathrm{f}}$ and $v_{\mathrm{f}}$ of $\mathrm{Pb}(111)$ film as a function film thickness from 1 to $30 \mathrm{MLs}$, respectively. Clearly, both $E_{\mathrm{f}}$ and $v_{\mathrm{f}}$ show a strong odd-even oscillation with a beating pattern period of $\sim 9$ MLs [19, 24], manifesting the QSE. Overall, both oscillation patterns are very similar to those of surface energy [24] and surface stress [19] of $\mathrm{Pb}(111)$ film. The oscillation amplitude for both $E_{\mathrm{f}}$ and $\nu_{\mathrm{f}}$ decays slowly and remains to be strong even for $\sim 30 \mathrm{ML}$ thick film. Figures 2(c) and (d) show the calculated $E_{\mathrm{f}}$ and $\nu_{\mathrm{f}}$ of $\mathrm{Al}(111)$ film from 1 to 29 MLs. Similar to $\mathrm{Pb}(111)$, strong oscillations are observed in both $E_{\mathrm{f}}$ and $\nu_{\mathrm{f}}$; different from $\mathrm{Pb}(111)$, the oscillations in $\mathrm{Al}(111)$ exhibit a three-fold periodic pattern, consistent with the previous findings in surface energy [25] and surface stress [20]. As shown by previous work, the reason for different primary QSE oscillation patterns in $\mathrm{Pb}(111)$ film (odd-even 2-layer oscillation) and $\mathrm{Al}(111)$ film (3-layer oscillation) is because the matching relationship between the electron Fermi wavelength and the film thickness in these two films are different. Apparently, these striking oscillations of elastic constants differ from the well-known SE that changes the elastic constants monotonically as a function of film thickness [8-13]. They clearly demonstrate the importance of QSE on modifying the elastic constants of nanofilms. For comparison, we next calculated the elastic constants of semiconductor $\mathrm{Si}(111)$ nanofilms, where QSE is known to be small. The calculated $E_{\mathrm{f}}$ and $\nu_{\mathrm{f}}$ of $\mathrm{Si}(111)$ films are shown from 6 to 14 MLs in figures 2(e) and (f), respectively. We did not include the data for films thinner than 5 ML because of the increasing nonlinear dependence of stress on strain as discussed in detail below for monolayer ribbon calculations. In contrast to $\mathrm{Pb}(111)$ and $\mathrm{Al}(111)$, the oscillations of $E_{\mathrm{f}}$ and $v_{\mathrm{f}}$ in $\mathrm{Si}(111)$ are much weaker, indicating a much smaller QSE in semiconductor $\mathrm{Si}(111)$ nanofilms.

To better understand the physical origin of quantum oscillations of elastic constants, we may consider a simple free electron gas model. The bulk modulus of a uniform electron gas of density $n$ is [26]

$$
B(n)=\frac{\hbar^{2}\left(3 \pi^{2}\right)^{2 / 3}}{3 m_{e}} n^{5 / 3} \propto n^{5 / 3} .
$$





Figure 3. Charge density distribution (Bardeen-Friedel oscillations) along $z$-direction of $\mathrm{Pb}$ (111) film. (a) $n_{\max }$ show the maximum charge density within each atomic planes; (b) $n_{\min }$ show the minimum charge density in between atomic planes; (c) $n_{\max }$ versus Pb film thickness; (d) $n_{\text {min }}$ versus $\mathrm{Pb}$ film thickness.

In a nanofilm, quantum confinement modulates the electron density along the surface normal $z$-direction. Figures 3(a) and (b) show the maximum charge density within an atomic plane $\left(n_{\max }\right)$ and the minimum density in between two atomic planes $\left(n_{\min }\right)$ along the $z$-direction in the $30 \mathrm{ML} \mathrm{Pb}(111)$ film, respectively. Clearly, both $n_{\max }$ and $n_{\min }$ exhibit the Bardeen-Friedel oscillations along the $z$-direction of the film, with the oscillation magnitude decaying away from the surface. Figures 3(c) and (d) shown $n_{\max }$ and $n_{\min }$ at the middle of the film as a function of film thickness, respectively, which exhibit an odd-even periodic oscillation originated from QSE. Thus, approximately, we apply equation (1) to the local density in the film, in the same spirit of local density approximation, and calculate the overall elastic modulus of the film as

$$
B_{\mathrm{f}}=\frac{1}{d} \int_{0}^{d} B(n, z) \mathrm{d} z \propto \frac{1}{d} \int_{0}^{d} n(z)^{5 / 3} \mathrm{~d} z .
$$

And the QSE modulated charge density leads to the QSE modulated film modulus and similarly other elastic constants.

Besides the QSE, the SE should be present also. If one considers the film has a thickness $(d)$ and bulk elastic constant $\left(C_{\mathrm{b}}\right)$, plus a surface layer of thickness $(\delta)$ and surface elastic constant $\left(C_{\mathrm{s}}\right)[12,13]$, the overall film elastic constant can be calculated as [11-13]

$$
C_{\mathrm{f}}=C_{\mathrm{b}}+\frac{2\left(C_{\mathrm{s}}-C_{\mathrm{b}}\right) \delta}{d}
$$

which shows an inverse linear dependence on the film thickness $(\sim 1 / d)$. Whether the surface becomes harder or softer depends on $C_{\mathrm{s}}$. If $C_{\mathrm{s}}>C_{\mathrm{b}}, C_{\mathrm{f}}$ increases with decreasing $d$; if $C_{\mathrm{s}}<$ $C_{\mathrm{b}}, C_{\mathrm{f}}$ decreases with decreasing $d$. Now, if we pretend to ignore the QSE and use equation (3) to forcefully fit the calculated $E_{\mathrm{f}}$ and $\nu_{\mathrm{f}}$, we got the dashed blue lines shown in figures 2(a)-(f), which roughly reflect the SE.

It is interesting to compare the results of metal film with those of semiconductor film, to reveal the relative importance of QSE versus SE. For $\mathrm{Pb}(111)$ film (figures 2(a) and (b)) the QSE modulation of elastic constants is so strong with an oscillation magnitude changing the elastic constants by $\sim 100 \%$, while the SE is less important changing the elastic constant by at most $\sim 26 \%$. For Al(111) film (figures 2(c) and (d)), the QSE modulation of elastic constants is also prominent comparing to $\mathrm{SE}$. In contrast, for $\mathrm{Si}(111)$ film (figures 2(e) and (f)), there is still QSE oscillations that modify the elastic constants on top of the SE, but they are relatively much smaller than in $\mathrm{Pb}(111)$ and $\mathrm{Al}(111)$ films. In particular the SE induces a change of Young's modulus of Si(111) film by $\sim 5 \%$ (figure 2(e)) while the QSE induced oscillation is insignificant. Therefore, we conclude that the QSE can be dominant in affecting the elastic constants of metal nanofilms overwhelming the SE, but plays a lesser role in affecting the elastic constants of semiconductor nanofilms. This is because the electron Fermi wavelength $\left(\lambda_{\mathrm{F}}\right)$ scales inversely with electron density $(n)$ in a power law $\left(\lambda_{\mathrm{F}} \propto n^{-1 / 3}\right.$ for $3 \mathrm{D}$ electron gas and $\lambda_{\mathrm{F}} \propto n^{-1 / 2}$ for $2 \mathrm{D}$ electron gas) [26], so that the Fermi wavelength is usually shorter in metals with a high electron density than in semiconductors with a low carrier density. Consequently, quantum confinement of electrons or carries, and hence the QSE is stronger in metal than in semiconductor. Second, the metal surfaces usually relax or reconstruct less than the semiconductor surfaces [3], so that the SE is expected to be weaker in metal nanostructures than in semiconductor nanostructures. 
(a)

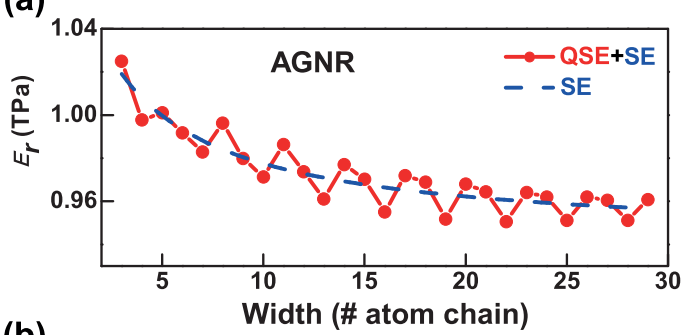

(b)

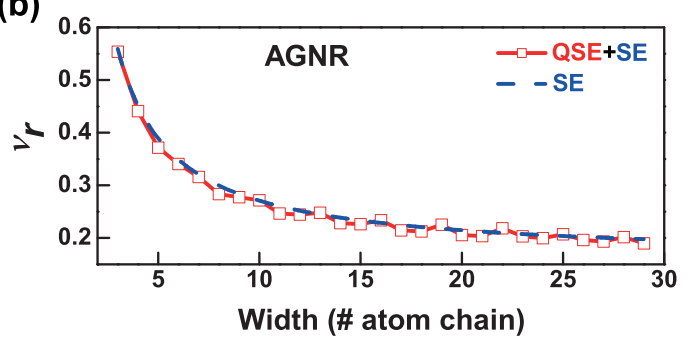

Figure 4. (a) Young's modulus and (b) Poisson's ratio of AGNR as a function of ribbon width. The dashed blue lines show the SE fitted using equation (3).

Besides nanofilms, QSE is expected commonly present in other low-dimensional metal nanostructures such as nanowires and quantum dots. A special case of low-dimensional nanostructures is GNR. In particular, strong QSE has been shown in armchair graphene ribbons (AGNRs), which is known to exhibit interesting electron band structure [27], with $1 / 3$ being metallic and $2 / 3$ being semiconducting in a three-atomic-raw oscillation as a function of ribbon width, as well as similar oscillations in edge energy and edge stress [17] (equivalent to surface energy and stress of nanofilm) induced by QSE. Figures 4(a) and (b) shows the calculated Young's modulus $\left(E_{\mathrm{r}}\right)$ and Poisson's ratio $\left(v_{\mathrm{r}}\right)$ of AGNR as a function of width from 3 to 29 atomic rows. Indeed, we see the quantum oscillations in both $E_{\mathrm{r}}$ and $v_{\mathrm{r}}$ induced by QSE, with a three-atomic-raw period similar to edge energy and edge stress [17]. We also fit the data using equation (3) to reveal the edge effect (the equivalent SE in 2D), shown as the red dashed line in figures 4(a) and (b). From the fitting, we obtained the graphene Young's modulus and Poisson's ratio to be $0.95 \mathrm{TPa}$ and 0.16 , in good agreement with previous work [28, 29]. For AGNR, the QSE induced oscillation magnitude is small changing the Young's modulus by a maximum of $2 \%$ and Poisson's ratio by $11 \%$, respectively, while the edge effect changes them by as much as $7 \%$ and $340 \%$, respectively, which indicates that, in AGNR, both edge effect and QSE effect are significant.

We note that our additional test calculations indicated that although the results of AGNRs show qualitatively the same behavior as nanofilms, they may not be simply generalized to other 2D monolayer systems. In particular, we found that the linear elastic constants of monolayer nanoribbons with buckling structures, such as silicene and $\mathrm{MoS}_{2}$ we tested, cannot be well-defined. This is because when in-plane (either uniaxial or biaxial) strain is applied, there is a large out-ofplane relaxation that changes the buckling angle, causing a nonlinear stress response even to a very small strain. In other words, the effective thickness of a buckled structure changes with strain, so that the elastic constants cannot be well-defined.
This is also the reason we did not include in figures 2(e) and (f) the results of $\mathrm{Si}(111)$ film less than $5 \mathrm{ML}$ (or equivalently viewed as multilayer silicene).

\section{Conclusions}

In summary, we have demonstrated that the QSE can have a profound effect in affecting the elastic constants of nanofilms, with interesting manifestation of size-dependent quantum oscillations, in addition to the monotonic size dependence induced by SE that has been widely recognized before. Most importantly, we show that for metal nanofilms the QSE induced oscillations can be the most dominant effect to completely overwhelm the SE. Our findings shed new light on our understanding of the mechanical properties of nanostructures by adding interesting quantum aspects to nanomechanics with broad implications, such as Nanoelectromechanical systems (NEMS), as the mechanical response of nanofilms to external stimuli could be modified if their elastic constants are modified by the QSE.

\section{Acknowledgments}

This work was support by DOE-BES (Grant No. DEFG0204ER46148) and NSF-MWN and Materials Theory program (Grant No. DMR-0909212). We also thank DOE-NERSC and the CHPC at University of Utah for providing the computing resources.

\section{References}

[1] Poncharal P, Wang Z L, Ugarte D and de Heer W A 1999 Science 2831513

[2] Cuenot S, Fretigny C, Demoustier-Champagne S and Nysten B 2004 Phys. Rev. B 69165410

[3] Liu F, Hohage M and Lagally M G 1999 Encyclopedia of Applied Physics (Surfaces and Interfaces, Structure of) ed H Immergut and G Trigg pp 321-52

[4] Liu F and Lagally M G 1996 Phys. Rev. Lett. 763156

[5] Zang J, Huang M and Liu F 2007 Phys. Rev. Lett. 98146102

[6] Villain P, Goudeau Ph, Renault P O and Badawi K F 2002 Appl. Phys. Lett. 814365

[7] Renault P O, Le Bourhis E, Villain P, Goudeau P, Badawi K F and Faurie D 2003 Appl. Phys. Lett. 83473

[8] Sun C T and Zhang H 2003 J. Appl. Phys. 931212

[9] Van Workum K and de Pablo J J 2003 Phys. Rev. E 67031601

[10] Miller R E and Shenoy V B 2000 Nanotechnology 11139

[11] Zhou L G and Huang H 2004 Appl. Phys. Lett. 841940

[12] Zang J and Liu F 2007 Nanotechnology 18405501

[13] Zang J and Liu F 2008 Appl. Phys. Lett. 92021905

[14] Schulter K F 1976 Surf. Sci. 55427

[15] Knight W D, Clemenger K, de Heer W A, Saunders W A, Chou M Y and Cohen M L 1984 Phys. Rev. Lett. 522141

[16] Liu F, Khanna S N and Jena P 1990 Phys. Rev. B 42976

[17] Huang B, Liu M, Su N, Wu J, Duan W, Gu B-L and Liu F 2009 Phys. Rev. Lett. 102166404

[18] Hu H, Liu M, Wang Z F, Zhu J, Wu D, Ding H, Liu Z and Liu F 2012 Phys. Rev. Lett. 109055501

[19] Liu M, Han Y, Tang L, Jia J-F, Xue Q-K and Liu F 2012 Phys. Rev. B 86125427 
[20] Flötotto1 D, Wang Z, Jeurgens L P H and Mittemeijer E J 2012 Phys. Rev. Lett. 109045501

[21] Kresse G and Hafner J 1993 Phys. Rev. B 47558

[22] Blochl P E 1994 Phys. Rev. B 5017953

Kresse G and Joubert J 1999 Phys. Rev. B 591758

[23] Perdew J P, Burke K and Ernzerhof M 1996 Phys. Rev. Lett. 773865

[24] Wei C M and Chou M Y 2002 Phys. Rev. B 66233408
[25] Han Y and Liu D J 2009 Phys. Rev. B 80155404

[26] Ashcroft N W and Mermin N D 1976 Solid State Physics (New York: Holt, Rinehart, and Winston)

[27] Yan Q, Huang B, Yu J, Zheng F, Zang J, Wu J, Gu B-L, Liu F and Duan W 2007 Nano Lett. 71469

[28] Kudin K N, Scuseria G E and Yakobson B I 2001 Phys. Rev. B 64235406

[29] Lee C, Wei X, Kysar J W and Hone J 2008 Science 321385 